\title{
Highly Birefringent Long Period Gratings Fabricated with Femtosecond Laser
}

\author{
Mykhaylo Dubov, Thomas D.P. Allsop, Amos Martinez, Vladimir Mezentsev, and Ian Bennion \\ Photonics Research Group, Aston University B4 7ET Birmingham, United Kingdom \\ m.dubov@aston.ac.uk
}

\begin{abstract}
Long Period Gratings (LPG) in standard fiber have been manufactured with a sharply focused near infrared (NIR) femtosecond laser beam. Polarization splitting of the attenuation bands is strongly dependent upon the inscription power.

(C2005 Optical Society of America

OCIS codes: 060.7140 Ultrafast processes in fibers ; 060.2340 Fiber optics components
\end{abstract}

\section{Introduction}

Since the early demonstrations of grating inscription in various fibers using a femtosecond laser [1], much effort has been spent to improve the quality of fiber gratings and to understand the mechanisms of the residual changes of the refractive index [2]. It was previously reported that a femtosecond post-processing of LPG fabricated by means of standard UV inscription results in a new type of fiber device - a directional bend sensor [3]. In this paper we demonstrate all-femtosecond technology for fabrication of LPG-based polarization sensitive fiber devices in a standard telecommunication fiber without prior photosensitization.

In our approach, we have used a sharp focusing x100 microscopic objective as opposed to typically used long focal distance optics. This allows for accurate positioning of the small modified area with respect to the fiber axis. Hence the controlled asymmetry is introduced in the resulting device. Further advantage is that the grating inscription and its post-processing are now combined in a single fabrication stage. Finally, the spectral stability against thermal annealing or under elevated temperatures was found to be much higher [4]. We have also found that asymmetrically fabricated LPGs have better sensitivity to bending at lower curvature $\left(0.3 \mathrm{~m}^{-1}\right)$ as compared to a standard UV-LPG in the same fiber $\left(2.0 \mathrm{~m}^{-1}\right)$. This suggests high polarization sensitivity of such gratings which is the main focus of the present paper.
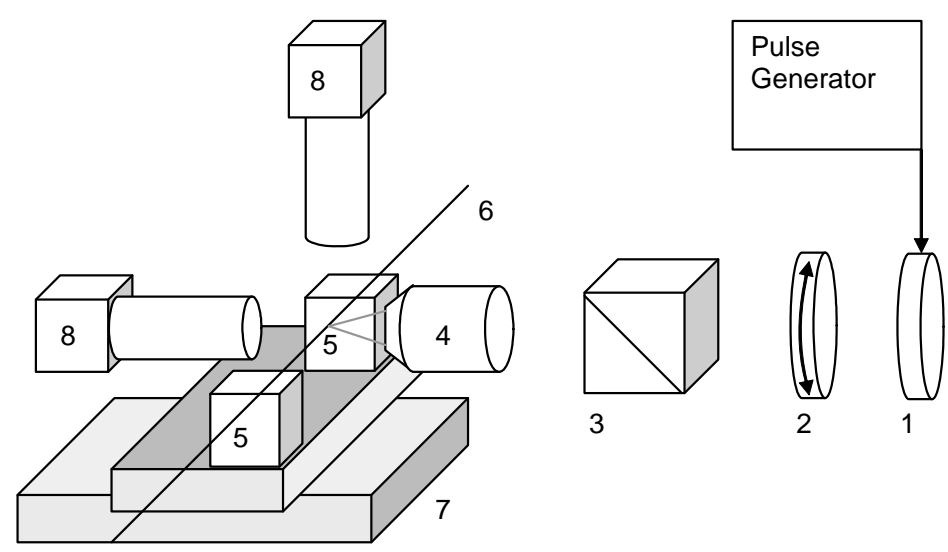

Figure 1. Optical layout for LPG inscription. The light passes a shutter (1), a half-wave plate (2), Glan prism (3), x100 long working distance microscopic objective (4), and is focused inside the fiber (6). Two alignment 3D-stages (5) are mounted on top of the high precision computer controlled 2D-stage (7).

\section{Experiment}

The schematic of the experimental set-up is presented in Fig.1. Laser system based on Spitfire Spectra Physics amplifier and Tsunami femtosecond mode-locked generator produces $120 \mathrm{fs}$ pulses at the wavelength of $796 \mathrm{~nm}$ at 1 $\mathrm{kHz}$ repetition rate. The computer controlled attenuation was realized with the half wave-plate (2) mounted on the motorised rotational stage, and a Glan prism (3). Electrical pulse generator was used to make rectangular pulses with a 50\% duty cycle to trigger the shutter (1). The standard SMF-28 fiber (6) was mounted on the computer controlled 
2D-stage (7) (ABL1000, Aerotech) and moved with a typical speed of $0.07 \mathrm{~mm}$ per second. Variation of the electrical pulse period and the fiber translation speed enabled fabrication of a series of LPGs with periods between 0.33 and $0.35 \mathrm{~mm}$. For the parameters mentioned above, it takes 5 seconds to inscribe one period (pitch) of the LPG. The inscription of the grating was done after the careful positioning of the focal spot with respect to the fiber core on both ends of the scanning range with the two auxiliary 3D-stages (5). We used a long working distance x100 objective (ApoSL, Mitutoyo) to focus the laser radiation. The power level was kept above the threshold of a supercontinuum appearance on the screen of the CCD-camera (8). As a result, a visible groove was typically produced inside the core of the fiber rather than a smooth track. Two CCD-cameras (8) with optical zooming systems were used for alignment and on-line monitoring of the inscription process.

A series of gratings with periods around $0.35 \mathrm{~mm}$ and a length of $45 \pm 5 \mathrm{~mm}$ have been made for the range of pulse energies. After the inscription, the microscopic images (Fig. 2) of all gratings were captured to check their positions in relation to the fiber axis. All gratings were offset by approximately the same distance from the centre of the core, as illustrated by Fig. 2.

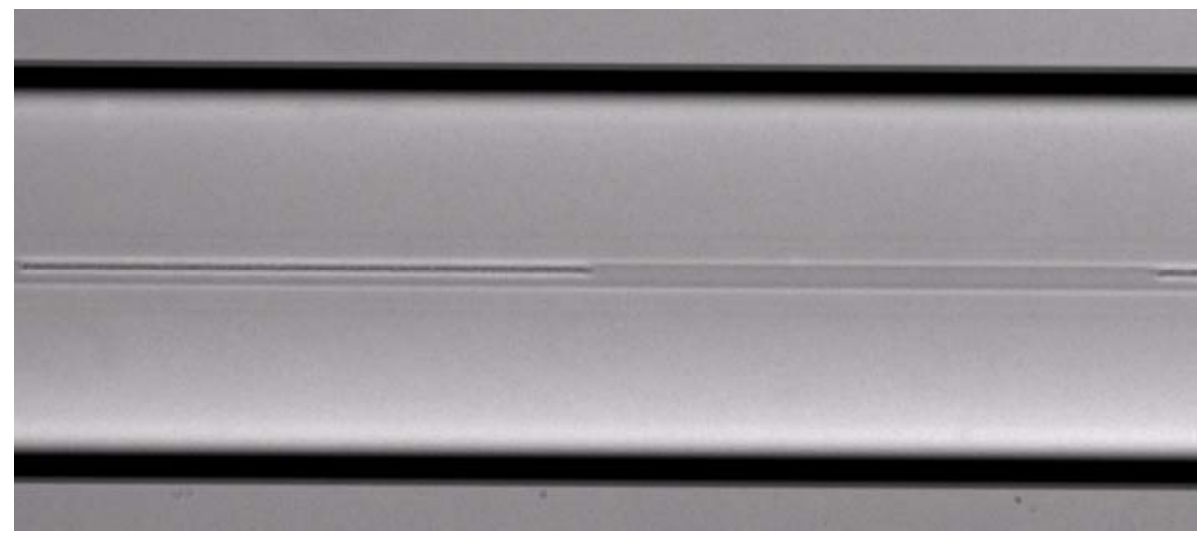

Figure 2. Microscopic image of the fiber with LPG. The groves are offset with respect to the core axis.

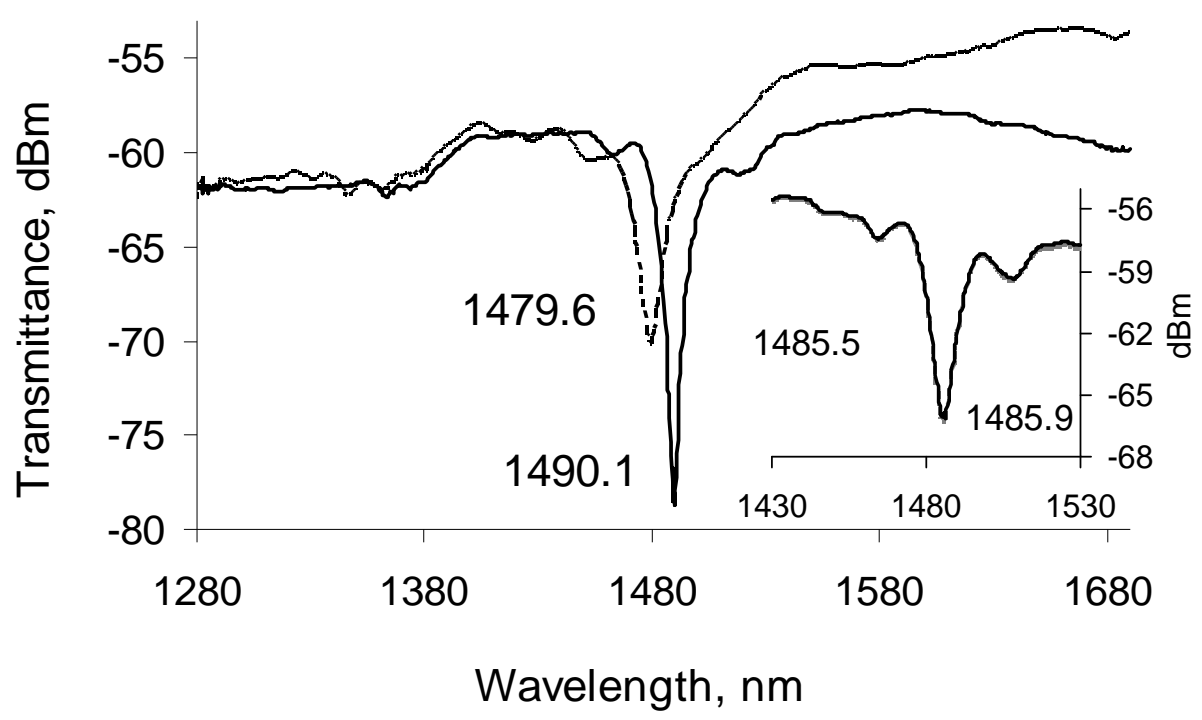

Figure 3. The transmission spectrum of the LPG made by the femtosecond laser. The positions of dips are marked on the graph. The insert shows the spectrum of the standard LPG made by a UV laser after hydrogenation.

The transmission spectrum of one of the LPGs made by the femtosecond inscription is shown in Fig.3. A typical spectrum of the standard UV inscribed LPG is shown in the insert for comparison. The transmission spectrum of the gratings is characterized by means of an optical spectrum analyzer with a $0.1 \mathrm{~nm}$ resolution. The light from the broadband source was launched into the grating under test after passing through the polarizer and a polarization 
controller. Figure 3 suggests, that the strength of the attenuation band for the long period grating written by the femtosecond laser is approximately $20 \mathrm{~dB}$, which is comparable to the UV inscribed LPG. Secondly, the LPG produced with femtosecond laser has a very strong dependence in the transmission dip positions for different polarizations $(10.5 \mathrm{~nm})$, compared with the standard UV-written LPG $(0.4 \mathrm{~nm})$. Another distinctive result (see Fig.4) was obtained when we checked the dependence in polarization splitting as a function of the pulse energy used for inscription. It is clear that femto-LPG tends to be more "birefringent" when higher pulse energy is used. The latter can be attributed to the stress induced by the femtosecond laser in the material, surrounding the modified area [2]. This modified structure is most likely to be a damaged waveguide. The visible size of the fabricated structure is less than the area affected by the laser, which could explain a big overlapping integral and strong attenuation bands. This was also confirmed by the thermal annealing results. Alternative explanation is that the perturbation of the waveguide, done by the femtosecond laser is not small, and the modal structure of the resulting waveguide is significantly different to that of the original fiber. Numerical simulations confirm a strong birefringence (the dependence of the effective refractive indexes for two orthogonal polarizations) even for a couple of fundamental modes.

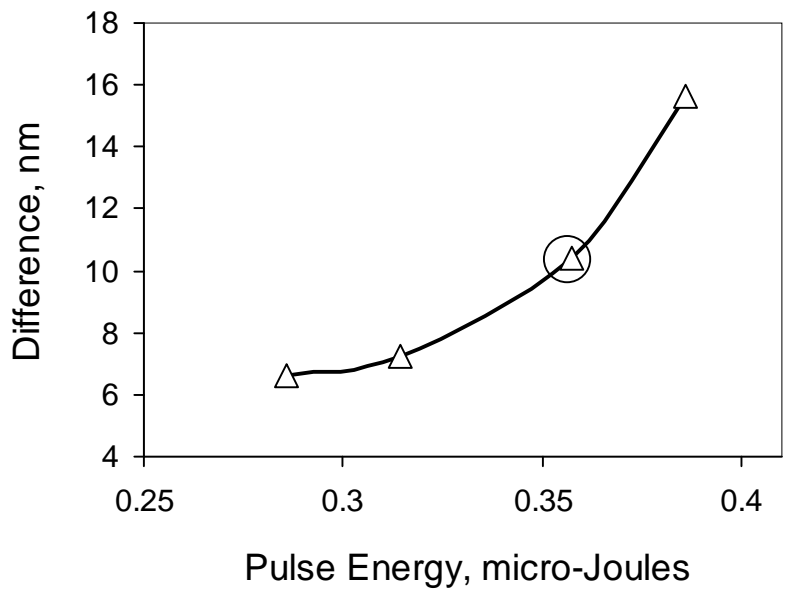

Fig.4. Dependence in polarisation splitting of the transmission dips vs. the energy of the pulse. The point marked with a circle corresponds to the femto-LPG from Fig.3.

\section{Conclusion}

In conclusion, we have produced strong long period gratings by sharply focused femtosecond laser pulses. These gratings reveal strong dependence in attenuation band positions and birefringence upon the polarization of light and the irradiation dose. Such polarization sensitive LPGs can be used in directional bend sensing, polarization controllers, fast switches and in a range of telecommunication applications.

\section{References}

[1]. A.Martinez, M.Dubov, I.Khrushchev and I.Bennion, “Direct writing of fibre Bragg gratings by femtosecond laser,” Electron.Lett. 40, 11701172 (2004).

[2] F. Dürr , H. G. Limberger, R. P. Salathe', F. Hindle, M. Douay, E. Fertein and C. Przygodzki, “Tomographic measurement of femtosecondlaser induced stress changes in optical fibers,” Appl.Phys.Lett. 84, 4983-85 (2004).

[3] T.Allsop, M.Dubov, A.Martinez, F.Floreani, I.Khrushchev, D.J.Webb, I.Bennion, "Long Period Grating Directional Bend Sensor Based Upon an Asymmetric Index Modification of Cladding,” Electron.Lett. 41, 59-60 (2005).

[4] A. Martinez, I.Y. Khrushchev and I. Bennion, "Thermal properties of fibre Bragg gratings inscribed point-by-point by infrared femtosecond laser," Electron.Lett. 41, 176-178 (2005). 